\title{
Transmission EBSD - Bridging the Gap between SEM and TEM
}

\author{
Amir Avishai ${ }^{1}$, Jennifer Carter ${ }^{2}$, Maryam Zahiri Azar², Badri Narayanan ${ }^{2}$, and Arthur H. Heuer ${ }^{1,2}$
}

1. Swagelok Center for Surface Analysis of Materials, Case Western Reserve University, Cleveland, OH, 44106

2. Dept. of Mat. Sci. and Eng., Case Western Reserve University, Cleveland, OH, 44106

With evermore powerful SEMs available on the market, the boundary between what could traditionally be accomplished using SEMs and TEMs is being continuously eroded. We have seen over the last several years the development of 3D reconstruction using FEG-SEMS with resolution approaching conventional TEM [1]. STEM imaging on SEM systems can achieve a resolution better than $7 \AA$ in all three modes (BF, DF and HAADF). This resolution capability, in conjunction with the ease of operation and versatility of the SEM, has made this a more accessible and useful system for materials research. The primary drawback of this approach has been the lack of crystallographic information to accompany the imaging data. In addition to already existing STEM imaging in various modes, we now have the ability to obtain fully automated transmission-EBSD maps on TEM samples in an SEM. This new platform makes it possible to conduct, with relative ease, in-depth experiments combining EDS mapping and crystallographic analysis with conventional imaging techniques [2-3]. One point that will have to be closely investigated is the volumes from which the information originates. However, this concern applies to combined EDS and EBSD in the SEM mode as well. The flexibility to load up to six samples with a standard FEI STEM holder in a Nova 200 Nano-lab, shown in Fig.1a, is a very useful, productive, and efficient aspect of the technique.

The new generation of integrated EDS-EBSD systems can acquire both chemical composition and crystallographic information as full 2D maps or (point measurements) simultaneously, and allows immediate identification of the crystal phase and orientation (Fig.1b). (We note that NanoMEGAS introduced the ASTAR system a few years ago for automated acquisition of diffraction information in TEM. It is still in its early stages, and is not yet widely available in modern TEMs). When chemical and crystallographic analysis is combined with dual beam FIB capability, a system is available that allows the user to prepare a site-specific TEM sample and completely characterize it in the SEM.

In this paper we will present examples of data obtained from different lift-out samples. We will examine the orientation relationship of an $\mathrm{Al}_{2} \mathrm{O}_{3}$ scale formed at high temperature on a Ni-base bond coat substrate, and chemical composition and crystal structure of a multi-component oxide slag. As the main example, a lift-out sample from a Ni-base super-alloy will be shown that was extracted from several grain boundaries that experienced significant strain accumulation, as had been determined by full field strain mapping [4]. TEM analysis and conventional EBSD analysis carried out on the same sample will also be presented, to demonstrate how multiple characterization techniques can be used for optimum data interpretation (see Fig. 2).

These recent developments have the potential to usher in a new generation of SEMs, equipped with fast position-sensitive detectors that can be inserted below the sample and will enable in the future extracting different signals ( $t$-EBSD, BF, DF, and HAADF) and obtaining the desired image, based on software integration of the signal, pixel by pixel, in different configurations. Another possible outcome may be 
microscopes with $40 \mathrm{kV}$ e-beams or higher on SEMs to obtain better resolution and the ability to work with thicker samples.

\section{References:}

[1] A Avishai et al., Microscopy and Microanalysis, 17, Supplement S2, June (2011), p 666.

[2] R R Keller and R H Geiss, Journal of Microscopy, 245 (2012), p. 245.

[3] P W Trimby, Ultramicroscopy, 120 (2012) p. 16.

[4] J L W Carter, et al., in Superalloys 2012, TMS, ed. E S Huron, (John Wiley \& Sons, Inc., Hoboken, New Jersey) (2012), p. 43.

[5] The authors would like to acknowledge the support of Rick Pessey from FEI and Scott Sitzman from Oxford Ins. for their help with the experimental setup and advice.
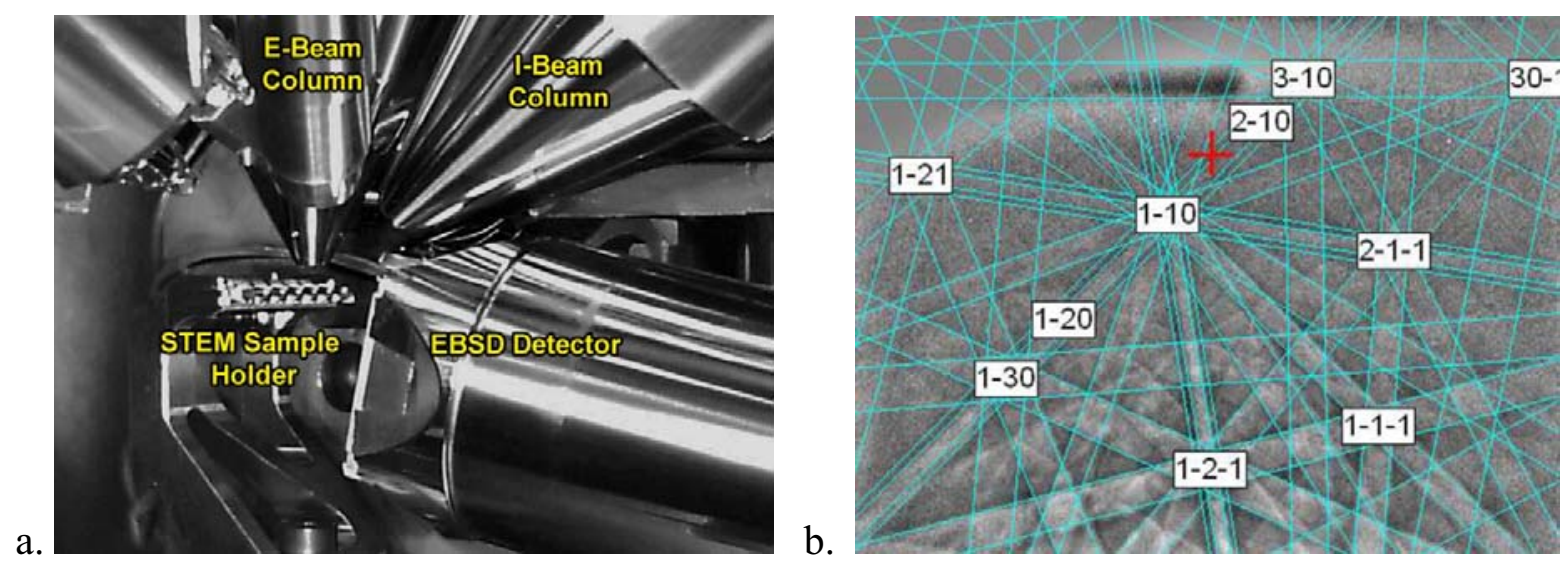

Figure 1. a) IR CCD camera image of the SEM chamber showing the STEM holder and transmission EBSD setup; b) Transmission EBSD Kikuchi pattern obtained from a Ni-base super-alloy overlaid with the crystallographic solution.
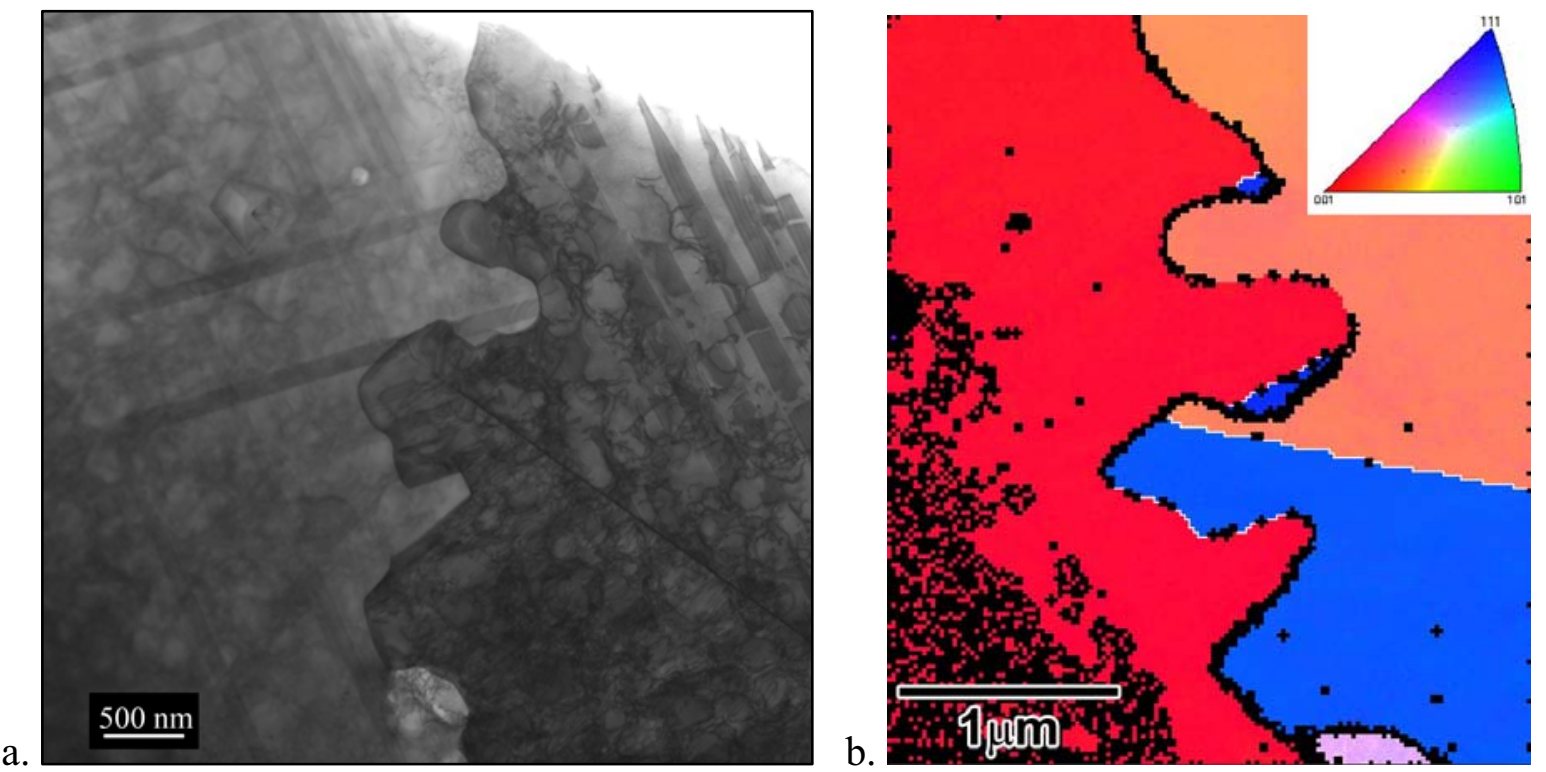

Figure 2. a) Bright Field STEM image, showing deformation twins in the left grain maybe the cause of the twinned regions near the grain boundary, b) Inverse pole figure map obtained from the same $\mathrm{Ni}$ based superalloy TEM sample with 20nm steps. 